\title{
16 DEVELOPMENT AND PILOT TESTING OF A NEW ACUTE PAEDIATRICS AND INJURY COURSE FOR AMBULANCE PROVIDERS IN KARACHI, PAKISTAN
}

doi:10.1136/injuryprev-2012-040590b.16

${ }^{1} \mathrm{~A}$ Chandran, ${ }^{2} \mathrm{G}$ Kazi, ${ }^{1} \mathrm{M}$ Eckerle, ${ }^{3} \mathrm{~S}$ Oureshi, ${ }^{1} \mathrm{AA}$ Hyder, ${ }^{2} \mathrm{~J}$ Razzak. ${ }^{1} \mathrm{~J}$ ohns Hopkins International Injury Research Unit, USA; ${ }^{2}$ Aga Khan Hospital, Pakistan; ${ }^{3}$ Aman Foundation, Pakistan

Background Appropriate and timely provision of pre-hospital care is critical for improving outcomes to injury and trauma. Pre-hospital providers in Pakistan as in most low and middle income countries do not receive standardised training in evidence-based acute paediatric care.

Aims/Objectives/Purpose Develop an Acute Paediatrics and Injury Care (APIC) course tailored to the local context and pilot test the course in ambulance providers in Karachi, Pakistan.

Methods APIC, a combination didactic and practical exercise-based course, was developed using validated international course materials and modifying them to the local context in collaboration with local paediatric and emergency providers. The course was piloted in 24 ambulance providers who were asked to complete pre and postcourse knowledge assessments and a 3 month follow up knowledge retention test.

Results/Outcome Upon course completion, students exhibited a statistically significant knowledge gain $(57.9 \%$ in the pre-test to $73.9 \%$ in the post-test, $p=<0.001$ ). Of the 19 students completing the knowledge retention test, students exhibited statistically significant gain in knowledge in the area of airway management/ respiratory distress $(56 \%$ to $74 \%, p=0.027)$, and a trend towards increased knowledge in shock ( $42 \%$ to $68 \%, p=0.06$ ), fluid administration $(74 \%$ to $95 \%, p=0.13)$ and trauma resuscitation $(74 \%$ to $84 \%, p=1.0)$.

Significance/Contribution to the Field The APIC course provided ambulance providers in Pakistan with important knowledge gains in paediatric-specific emergency care, particularly in the area of airway management and respiratory distress. Further studies are needed for expansion to a wider audience and to assess the impact of such courses on actual outcomes of paediatric illness, injuries and trauma. 